International Journal of Bifurcation and Chaos, Vol. 19, No. 2 (2009) 737-743

(C) World Scientific Publishing Company

\title{
SPATIOTEMPORAL COHERENCE RESONANCE IN A MAP LATTICE
}

\author{
XIAOJUAN SUN ${ }^{*} \dagger$ and QISHAO LU ${ }^{\ddagger}$ \\ School of Science, Beihang University, \\ 100083 Beijing, P. R. China \\ †sxiaojuan@gmail.com \\ ${ }^{\ddagger}$ qishaolu@hotmail.com \\ JÜRGEN KURTHS \\ *Institute of Physics, University of Potsdam, \\ Potsdam Institute for Climate Research \\ PF 601203, 14412 Potsdam, Germany \\ juergen.kurths@pik-potsdam.de \\ QINGYUN WANG \\ Inner Mongolia Finance and Economics College, \\ 010051 Huhhot, P. R. China \\ nmqingyun@163.com
}

Received March 14, 2008; Revised May 26, 2008

\begin{abstract}
We study the effects of parametric noise on a lattice network, which is locally modeled by a twodimensional Rulkov map. We conclude that at some intermediate noise intensity, parametric noise can induce ordered circular patterns, which indicates the appearance of spatiotemporal coherence resonance in the studied lattice. With the observation of coherence-like manner in linear spatial cross-correlation, the coherence phenomena can be analyzed quantitatively.
\end{abstract}

Keywords: Noise; coupled map lattices; pattern formation.

\section{Introduction}

It is a well-documented fact that noise can induce temporal resonance in different kinds of nonlinear dynamical systems [Gammaitoni et al., 1998]. In coherence resonance [Pikovsky \& Kurths, 1997] or autonomous stochastic resonance [Longtin, 1997], pure noise without an external signal can generate the most coherent motion in systems, as has been mainly observed in excitable systems [Pikovsky \& Kurths, 1997; Longtin, 1997; Lee et al., 1998; Hu et al., 1993]. Recently, the standard temporal coherence resonance has been extended and studied in many coupled dynamical systems [Lindner et al., 1995; Hu \& Zhou, 2000; Zhou et al., 2001; Carrillo et al., 2004; Perc, 2005, 2007; Sun et al.,
2008]. Coupled enhancing coherence has been found in array coupled systems, which is called array enhanced coherence resonance (AECR) in [Lindner et al., 1995] and [Hu \& Zhou, 2000]. Moreover, it has been further found that the inhomogeneity in the parameters of the array enhances AECR [Zhou et al., 2001]. Spatial coherence resonance has already been discussed in different nonlinear media (chlorine dioxide-iodine-malonic acid reaction [Carrillo et al., 2004]; Rulkov map [Perc, 2007]; FitzHugh-Nagumo [Perc, 2005]; HodgkinHuxley neuronal model [Sun et al., 2008]). Without any other external excitations, noise can extract an intrinsic spatial scale optimally in the media [Carrillo et al., 2004] and a characteristic spatial 
frequency of the systems in a resonant manner [Perc, 2005, 2007; Sun et al., 2008].

The ability of noise to enhance spatiotemporal patterns and to increase some coherence or collective dynamical properties may be viewed as a generalization of stochastic resonance to spatially extended systems, named as spatiotemporal stochastic resonance (STSR) or spatiotemporal coherence resonance (STCR) if there is no other external excitations except for noise. This spatiotemporal coherence has been reported in many articles in the past years [Jung \& MayerKress, 1995; Hou et al., 1998; Zhou \& Kurths, 2002; Hütt et al., 2002; Busch \& Kaiser, 2003]. Jung and Mayer-Kress [1995] numerically observed STSR in excitable media, while Zhou and Kurths [2002] demonstrated STCR in chaotic oscillatory media. Busch and Kaiser [2003] used the spatial cross-correlation, which is similar to the method developed for detecting signatures of noise-induced structures in spatiotemporal data sets [Hütt et al., 2002] when the noise intensity is not known experimentally, to discuss the STSR phenomena induced by spatiotemporally correlated colored noise.

In this paper, we study the influence of parametric noise on spatiotemporal patterns of a square lattice localized by the Rulkov map [Rulkov, 2001, 2002] (therefore, the noise is coupled with the system in a multiplicative way). With the aid of spatial cross-correlation [Busch \& Kaiser, 2003], we detect STCR that occurs in the lattice.

The rest of this paper is organized as follows: in Sec. 2, we give the equations of the square lattice networks. In Sec. 3, we study the effects of parametric noise on pattern formations and the noise-induced spatiotemporal coherence resonance. Finally, we present the conclusion and discussion in Sec. 4.

\section{Equations of the Square Lattice Networks}

The model equations of this spatially extended system (lattice) is given as follows:

$$
\left\{\begin{aligned}
u_{n+1}(i, j)= & \frac{\left(\alpha+\xi_{n}(i, j)\right)}{\left(1+u_{n}^{2}(i, j)\right)}+v_{n}(i, j) \\
& +D\left(u_{n}(i+1, j)+u_{n}(i-1, j)\right. \\
& +u_{n}(i, j-1)+u_{n}(i, j+1) \\
& \left.-4 u_{n}(i, j)\right), \\
v_{n+1}(i, j)= & v_{n}(i, j)-\beta u_{n}(i, j)-\gamma,
\end{aligned}\right.
$$

where the pair $(i, j)$ indicates that the neuron is at the $i$ th unit in the horizontal and the $j$ th unit in the vertical direction, $i, j=1, \ldots, N$. The subscript $n$ is the discrete time index, and $D$ is the diffusion coefficient. $u_{n}$ is the membrane potential of the neuron and $v_{n}$ is the variation of ion concentration. $u_{n}, v_{n}$ represent the fast and slow dynamical variables respectively. $\alpha, \beta, \gamma$ are system parameters and $\beta, \gamma$ are both taken as 0.001. For $\alpha<2.0$, the uncoupled single unit in this lattice is governed by a single excitable steady state $(-1,-1-\alpha / 2)$. Here we take $\alpha$ as 1.99 . For a more detailed analysis of the dynamical behavior of the single Rulkov map, refer to [Rulkov, 2001, 2002]. Each unit is subject to a multiple Gaussian white noise $\xi_{n}(i, j)$, which is uncorrelated both in discrete time and space, namely

$$
\left\langle\xi_{n}(i, j) \xi_{m}(k, l)\right\rangle=2 \sigma \delta_{n, m} \delta_{i, k} \delta_{j, l},
$$

where $\sigma$ is the noise intensity. At each iterated step for each unit $(i, j)$ random numbers $\xi_{n}(i, j)$ are generated by the Box-Mueller algorithm [Fox et al., 1988].

In this paper, we take $D, \sigma$ as control parameters and fix $N=128$. We study the effects of the noise intensity $\sigma$ on spatiotemporal pattern formation for various diffusion coefficients $D$.

\section{Spatiotemporal Coherence Resonance}

The influences of the parametric noise on spatiotemporal pattern formation of the lattice [Eq. (1)] are depicted in the contour plots in Fig. 1. For each particular diffusion coefficient $D$, there exist intermediate noise intensities for which we can perceive coherent spatial structures, i.e. circular waves (middle column of Fig. 1). For smaller and larger $\sigma$ at each particular $D$, the noise-induced patterns do not show any visible spatial structures. For smaller noise, the noise intensities are not strong enough to excite the system to evoke any particularly outstanding spatial structures, as shown in the left column of Fig. 1; for larger noise, ordered circular waves are broken down by strong noisy perturbations, yielding disordered looking spatial portraits, as presented in the right column of Fig. 1. Such spatial patterns formed for the intermediate noise intensities are typically observed also in pattern formation of deterministic systems ([Arecchi et al., 1999] and references therein).

In order to quantify the formation of those structures, we introduce a linear spatial cross-correlation measure $S$. We compute the 

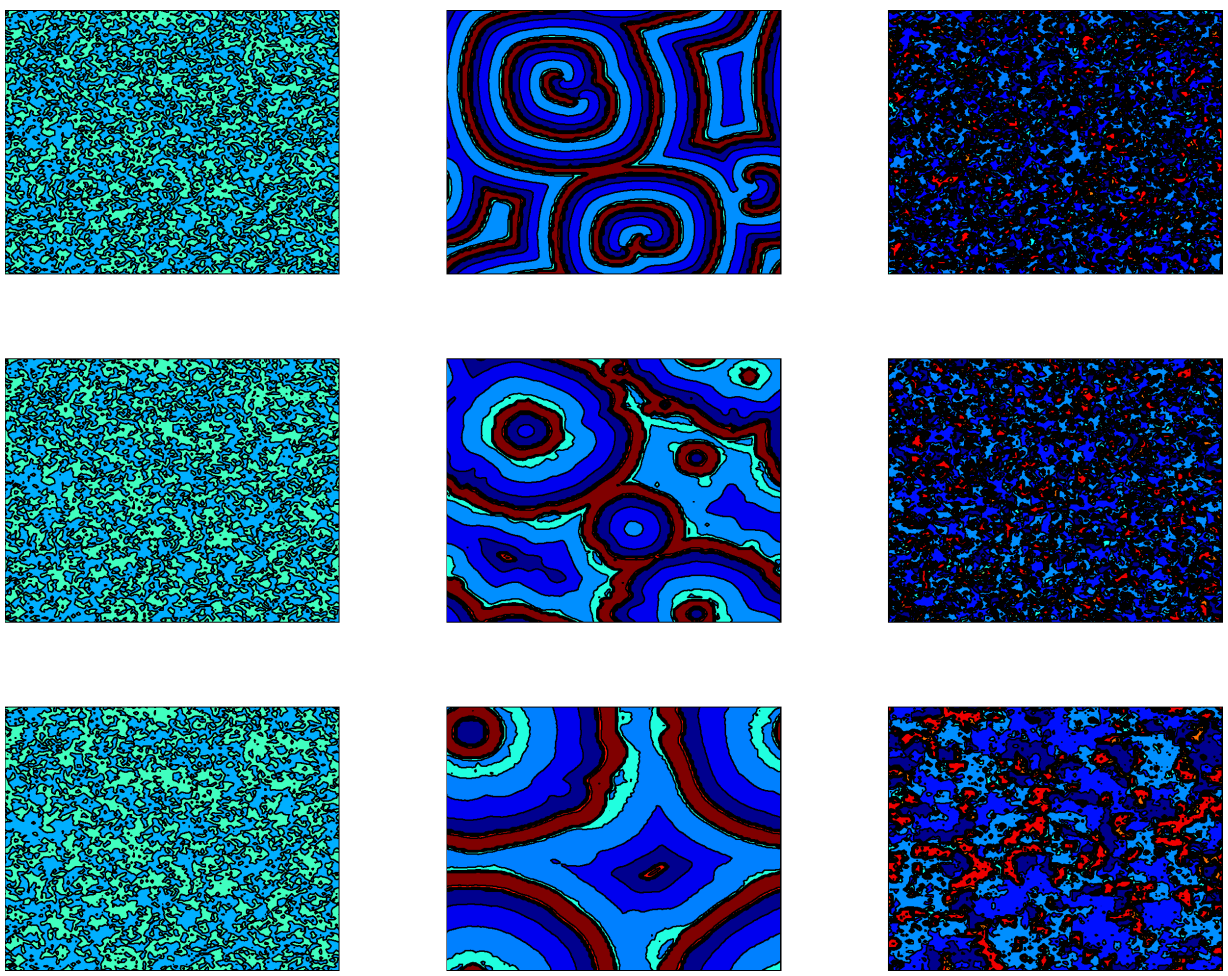

Fig. 1. Snapshots of the lattice for various noise intensity $\sigma$ and diffusive coupling strength $D$. Shown are the $u(i, j)$ variables at each grid point. From top to bottom at each row, $D$ is taken as $0.0025,0.005,0.01$ respectively. For each $D, \sigma$ increases from left to right. For $D=0.0025, \sigma=1 E-7,3 E-6,1 E-3$, for $D=0.005, \sigma=1 E-6,4 E-6,2 E-3$, and for $D=0.01$, $\sigma=4 E-6,8 E-6,2 E-3$. All the snapshots of the spatial grid are taken at given times $n$.

cross-correlation $S$ for the variable $u_{i, j}$ as the space and time averaged nearest-neighbor distance of all elements, normalized by the total spatial amplitude variance. $S$ is given by

$$
S=\left\langle\frac{\operatorname{Cov}(n)}{\operatorname{Var}(n)}\right\rangle_{T}
$$

where the bracket \langle\rangle$_{T}$ denotes averaging over the total iterated time $T$. $\operatorname{Var}(n)$ is the spatial variance at the iterated time $n$ given as

$$
\operatorname{Var}(n)=\frac{1}{N^{2}} \sum_{i j}(u(i, j)-\bar{u})^{2}
$$

where $\bar{u}=N^{-2} \sum_{i j} u_{i, j} ; \operatorname{Cov}(n)$ is the purely spatial auto-covariance of nearest neighbors, and is defined as

$$
\operatorname{Cov}(n)=\frac{1}{N^{2}} \sum_{i j} \frac{1}{\left|\mathcal{N}_{i, j}\right|} \sum_{b \in \mathcal{N}_{i j}}(u(i, j)-\bar{u})(b-\bar{u})
$$

with $b$ consisting of all $\left|\mathcal{N}_{i j}\right|=4$ elements of a von Neumann neighborhood $\mathcal{N}_{i j}$ at each lattice site $u(i, j)$. Obviously, the quantity $S$ is efficient in analyzing nearest-neighbor relationships in space and time. The larger the value of $S$ is, the more coherent the pattern becomes.

Figure 2(a) shows $S$ as a function of the noise intensity $\sigma$ for various diffusion coefficients $D$. We observe that for each $D, S$ increases with increasing $\sigma$ at the beginning, then it reaches a maximum value, and after that it begins to decrease if $\sigma$ increases again. This resonance-type behavior in $\sigma$ for each $D$ indicates the signature of spatiotemporal coherence resonance. Furthermore, we can see that after the patterns fire, i.e. some neurons in the lattices are firing, the larger $D$ corresponds to the larger $S$ [see Fig. 2(b)]. It means that the spatial structures at larger diffusion coefficients are more coherent than those at smaller ones. Since a larger $D$ can make local excitations propagate farther and the units of the whole lattice are more connected to each other, the coherence of the whole lattice increases with the increase of $D$. From Fig. 2(a), we find that the spatial cross-correlation $S$ takes up the same values for smaller and larger noise intensities, i.e. the patterns at smaller and larger $\sigma$ have the same coherence. But we know that the spatiotemporal patterns have quite different behaviors for these two cases (one is caused by firing but the 


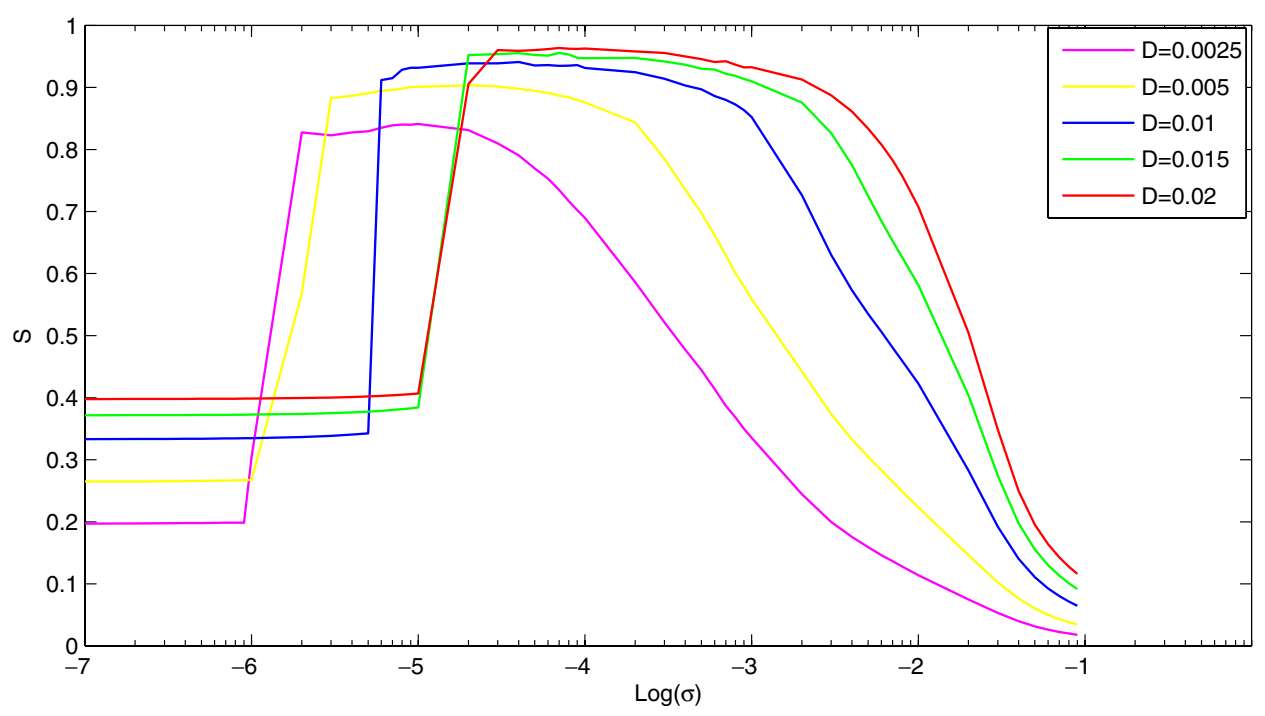

(a)

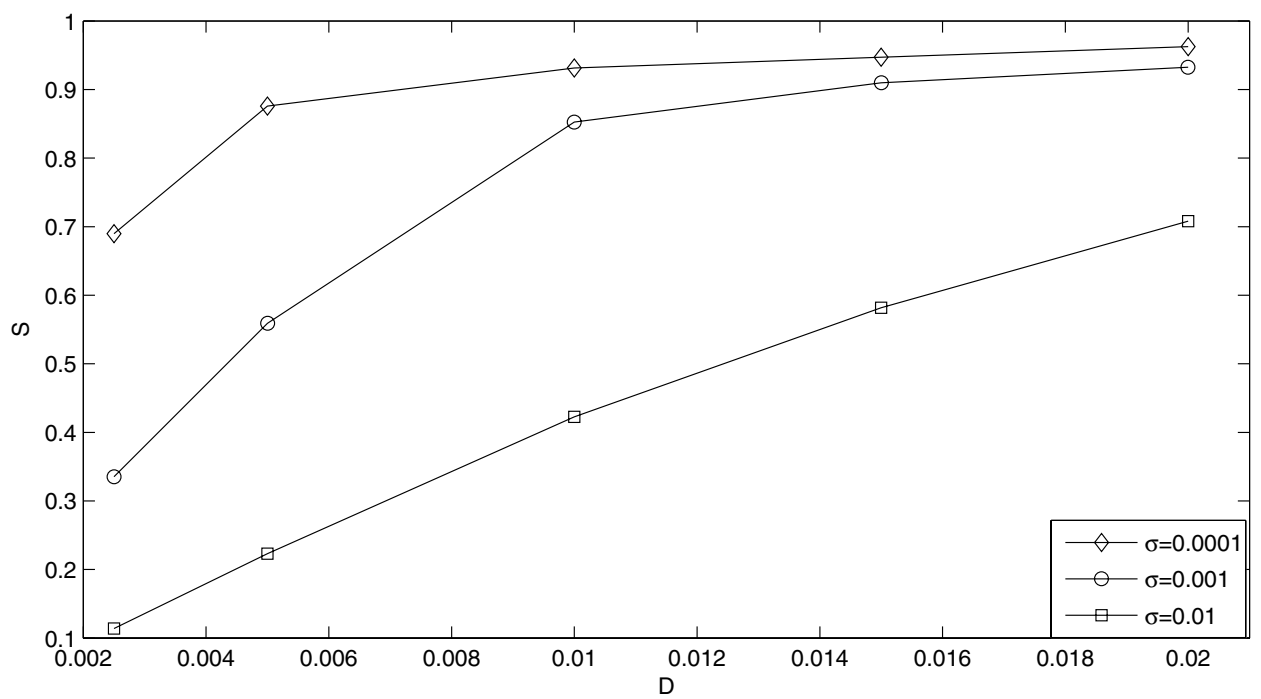

(b)

Fig. 2. Spatial cross-correlation $S$ (a) $S$ versus noise intensity $\sigma$ in log scale at various diffusion coefficients $D$; (b) $S$ versus diffussion coefficient $D$ for various noise intensities $\sigma$. The line is just a guide to the eye.

other is not; see Fig. 1). So we should introduce a new quantity to distinguish these two cases and to analyze the mechanism of the numerically observed STSR that occurred in the lattice [Eq. (1)].

The quantity we introduce is the averaged firing-rate function $\langle\Pi(n)\rangle_{T}$, where the bracket \langle\rangle$_{T}$ denotes the average of total iterated time $T$. The equation of the firing-rate function $\Pi(n)$ is given as

$$
\Pi(n)=\frac{m(n)}{N^{2}} .
$$

When the membrane potential of a single Rulkov map reaches the threshold value $u_{\text {th }}=-0.2$ from below at a given time, we say that this map is firing. $m(n)$ indicates that $m$ neurons are firing simultaneously at a given iterated time $n$. If $m(n)>0$, some neurons are firing at the given time $n$; otherwise, all neurons will fluctuate around its stable excited state. Figure 3 shows $\langle\Pi(n)\rangle_{T}$ as a function of the noise intensity (in a log scale) for various $D$ respectively corresponding to Fig. 2(a). It can be clearly seen that for smaller noise intensities, $\langle\Pi(n)\rangle_{T}=0$, i.e. the neurons are not firing at all. While for larger noise intensities, $\langle\Pi(n)\rangle_{T}>0$, i.e. some of the neurons in the lattice are firing due to parametric noise. Thus, with 


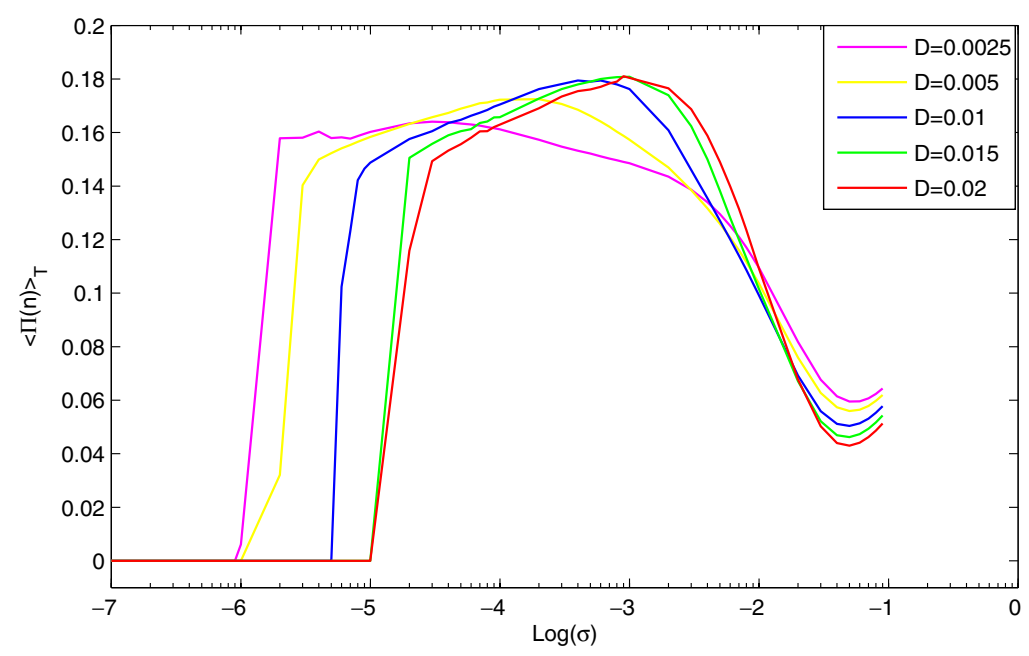

Fig. 3. Averaged firing rate function $\langle\Pi(n)\rangle_{T}$ versus diffusion coefficient $D$ corresponding to Fig. 2(a).

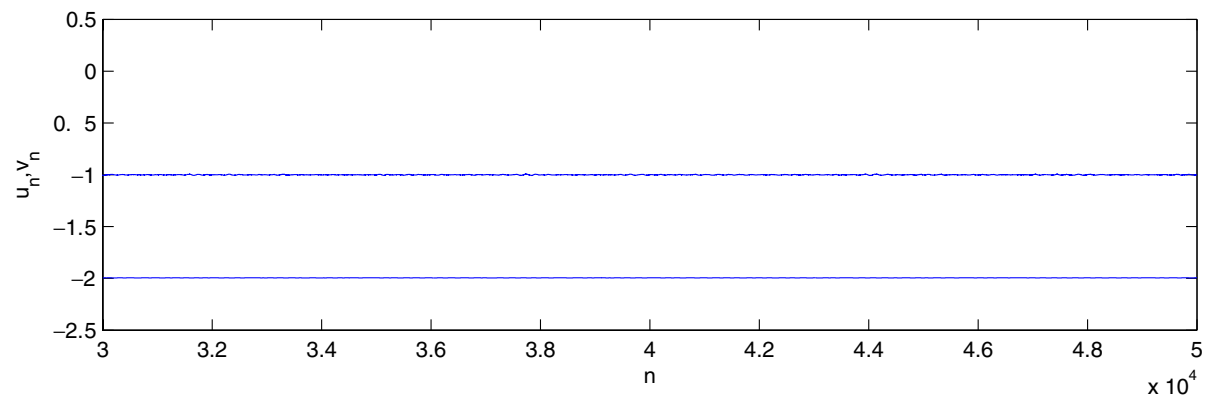

(a)

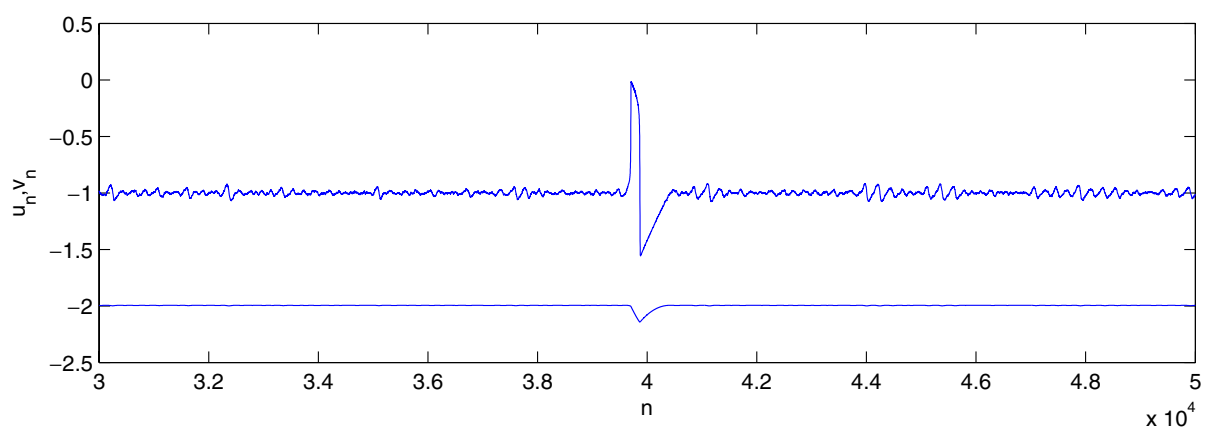

(b)

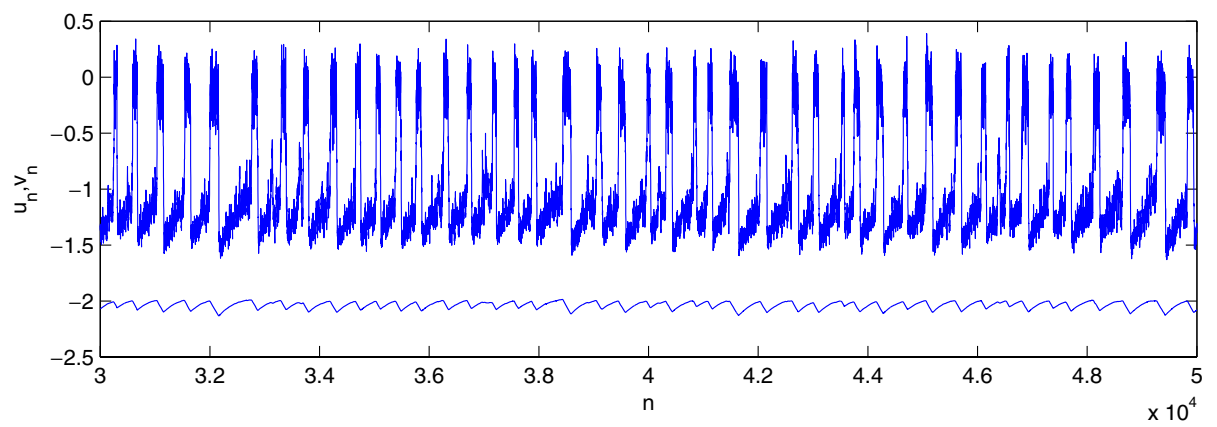

(c)

Fig. 4. Noise-induced oscillations in a single unit of the lattice. (a) $\sigma=1 E-7$, (b) $\sigma=7 E-6$ and (c) $\sigma=1 E-2$. 
the introduction of the averaged firing rate function $\langle\Pi(n)\rangle_{T}$, we can successfully distinguish these two cases.

In the following, we intend to analyze the mechanism of STCR that occurred in the lattice [Eq. (1)]. We plot the waveforms of a single unit in this lattice for three different $\sigma$ (Fig. 4). For smaller $\sigma$, it just fluctuates around its excitable steady state [Fig. 4(a)], i.e. all units in the lattice cannot be firing, namely $\langle\Pi(n)\rangle_{T}=0$ as shown in Fig. 3. Thus, there is no nucleus and the lattice cannot generate any pattern formation (left column of Fig. 1). Hence, the coherence is small. When $\sigma$ increases a bit to $7 \times 10^{-6}$, the system generates sparse spikes [Fig. 4(b)]. For a larger noise intensity, a particular unit in the lattice can first fire at some iterated time $n$. Then it becomes a circular front initiator and the waves can spread to fire the other units. After some iterated times, the waves can spread through the whole lattice and the patterns exhibit circular spatial profiles (middle column of Fig. 1), and the coherence of the profiles becomes larger. When the noise intensity $\sigma$ continues to increase to larger values, the system is able to generate dense spikes from the beginning of iteration as shown in Fig. 4(c). It follows that all units in the lattice can be firing due to parametric noise at the beginning. These firing units all have the desire to spread. It will lead to a competition amongst them, which makes the waves of the units unable to spread further. Moreover, it makes the amount of the firing unit decrease (see Fig. 3). Therefore, the coherence of the patterns (as shown in the right column of Fig. 1) decreases at larger $\sigma$. With the above analysis, we understand how the STCR is created in the lattice.

\section{Summary and Conclusions}

In this paper, we study the influences of parametric noise on spatiotemporal patterns of a spatially extended system, which is locally modeled by the Rulkov map. From our numerical results, we can see that parametric noise is able to induce spatiotemporal coherence resonance, which is indicated by a maximum value of $S$ and ordered circular pattern structures.

We know that the two-dimensional Rulkov map replicates the dynamics of spiking and spikingbursting activity of real biological neurons. So the results we obtained in this paper may have some potential implications for understanding signal or information transition in real neuronal systems.
In nature, the units in a network are not identical. In [Glatt et al., 2007] and [Gassel et al., 2007], it is found that parameter variability in local systems can induce coherent patterns in a lattice. But the localized system used in these two papers is a continuous system. So we will next extend our study to lattices localized by nonidentical Rulkov maps. We expect that STCR could also be observed in such a lattice.

\section{Acknowledgments}

Q. S. Lu and X. J. Sun are grateful to the support of National Natural Science Foundation of China No. 10432010. Q. Y. Wang and X. J. Sun are grateful to the support of National Natural Science Foundation of China No. 10702023. J. Kurths is grateful to the support of SFB 555(DFG).

\section{References}

Arecchi, F. T., Boccaletti, S. \& Ramazza, P. [1999] "Pattern formation and competition in nonlinear optics," Phys. Rep. 318, 1-83.

Busch, H. \& Kaiser, F. [2003] "Influence of spatiotemporally correlated noise on structure formation in excitable media," Phys. Rev. E 67, 041105.

Carrillo, O., Santos, M. A., García-Ojalvo, J. \& Sancho, J. M. [2004] "Spatial coherence resonance near pattern-forming instabilities," Europhys. Lett. 65, $452-458$.

Fox, R. F., Gatland, I. R., Roy, R. \& Vemuri, G. [1988] "Fast, accurate algorithm for numerical simulation of exponentially correlated colored noise," Phys. Rev. A 38, 5938-5940.

Gammaitoni, L., Hänggi, P., Jung, P. \& Marchesoni, F. [1998] "Stochastic resonance," Rev. Mod. Phys. 70, 223-287.

Gassel, M., Glatt, E. \& Kaiser, F. [2007] "Doubly diversity-induced resonance," Phys. Rev. E $\mathbf{7 6}$, 016203.

Glatt, E., Gassel, M. \& Kaiser, F. [2007] "Variabilitysustained pattern formation in subexcitable media," Phys. Rev. E 75, 026206.

Hou, Z. H., Yang, L. F., Zuo, X. B. \& Xin, H. W. [1998] "Noise induced pattern transition and spatiotemporal stochastic resonance," Phys. Rev. Lett. 81, 28542857.

Hu, B. B. \& Zhou, C. S. [2000] "Phase synchronization in coupled nonidentical excitable systems and array-enhanced coherence resonance," Phys. Rev. E 61, R1001.

Hu, G., Ditzinger, T., Ning, C. Z. \& Haken, H. [1993] "Stochastic resonance without external periodic force," Phys. Rev. Lett. 71, 807-810. 
Hütt, M.-Th., Neff, R., Busch, H. \& Kaiser, F. [2002 "Method for detecting the signature of noise-induced structures in spatiotemporal data sets," Phys. Rev. E 66, 026117.

Jung, P. \& Mayer-Kress, G. [1995] "Spatiotemporal stochastic resonance in excitable media," Phys. Rev. Lett. 74, 2130-2133.

Lee, S.-G., Neiman, A. \& Kim, S. [1998] "Coherence resonance in a Hodgkin-Huxley neuron," Phys. Rev. E 57, 3292-3297.

Lindner, J. F., Meadow, B. K., William, L. D., Inchiosa, M. E. \& Bulsara, A. R. [1995] "Array enhanced stochastic resonance and spatiotemporal synchronization," Phys. Rev. Lett. 75, 3-6.

Longtin, A. [1997] "Autonomous stochastic resonance in bursting neurons," Phys. Rev. E 55, 868-876.

Perc, M. [2005] "Spatial coherence resonance in excitable media," Phys. Rev. E 72, 016207.

Perc, M. [2007] "Spatial coherence resonance in neuronal media with discrete local dynamics," Chaos Solit. Fract. 31, 64-69.
Pikovsky, A. S. \& Kurths, J. [1997] "Coherence resonance in a noise-driven excitable system," Phys. Rev. Lett. 78, 775-778.

Rulkov, N. F. [2001] "Regularization of synchronization chaotic bursts," Phys. Rev. Lett. 86, 183-186.

Rulkov, N. F. [2002] "Modeling of spiking-bursting neural behavior using two-dimensional map," Phys. Rev. E 65, 041922.

Sun, X. J., Perc, M., Lu, Q. S. \& Kurths, J. [2008] "Spatial coherence resonance on diffusive and smallworld networks of Hodgkin-Huxley neurons," Chaos 18, 023102.

Zhou, C. S., Kurths, J. \& Hu, B. B. [2001] "Arrayenhanced coherence resonance: Nontrivial effects of heterogeneity and spatial independence of noise," Phys. Rev. Lett. 87, 098101.

Zhou, C. S. \& Kurths, J. [2002] "Spatiotemporal coherence resonance of phase synchronization in weakly coupled chaotic oscillators," Phys. Rev. E 65, 040101(R). 